\title{
Observer Based LQ Optimal Boundary Control of 2D Heat Flow by Order Reduction
}

\author{
Mehmet Önder Efe
}

\begin{abstract}
Linear Quadratic (LQ) optimal boundary control of a 2D heat flow is studied. The design is carried out on a reduced order model of the Partial Differential Equation (PDE) process. For this purpose, Proper Orthogonal Decomposition (POD) technique is utilized and the Low Dimensional (LD) model is derived. The boundary controller is developed using the state information obtained via an observer. An infinite dimensional version of the observer is developed first and its finite dimensional counterpart is derived according to POD procedure. Having obtained the states of the system, a LQ optimal control approach is followed to demonstrate that the entire design works satisfactorily under the presence of noise, uncertainty and disturbances. The contribution of the paper is to draw a clear path between a spatially continuous process and an optimal boundary controller minimizing a quadratic cost, and the emphasis on the merits of POD based designs.
\end{abstract}

\section{INTRODUCTION}

Many physical processes such as heat and fluid flows, chemical processes and those involving mass and energy flows over a spatial domain are characterized by PDEs. The desire for maintaining a good level of a set of performance measures motivates the design and implementation of a feedback controller. Since the process is spatially continuous (infinite dimensional) one might design an infinite dimensional controller then reduce it, or one might reduce the process then design a controller. This paper adopts the latter, i.e. the process undergoes an order reduction procedure called POD, a LD observer is designed then, and a feedback controller based on a LD observer is derived utilizing the standard tools of control theory.

In this paper the cost function is a quadratic one and is defined over an infinite horizon. The difficulty that needs to be alleviated is the noise corrupting the observations from the infinite dimensional process. An observer is developed to filter out the disturbances from process state, which is once obtained, the designer can implement an appropriate LQ optimal controller in finite dimensions.

Optimal control is one of the frequently used approaches in boundary control applications of PDE systems. One work focusing on 1D heat conduction problem reports the design of time-optimal boundary control, [1], with the emphasis that the time-optimal control has the bang-bang property, and the solution has been postulated by the techniques of Hilbert spaces. In [2], Rösch views the characterization of boundary condition as an identification problem, and presents

This work was supported by TOBB Economics and Technology University, BAP Program, under contract no ETÜ-BAP-2006/04

Dr. Efe is with TOBB Economics and Technology University, Department of Electrical and Electronics Engineering, Sögütözü, Ankara, Turkey, Phone: +90-312-292-4064, Fax: +90-312-292 4180 onderefedieee. org an iterative approach to meet the conditions of optimality. Ravindran [3] and Singh et al [4] follow the optimal control techniques on more complicated flow systems, namely a flow past a step in [3] and flow past a cylinder in [4]. Both of these works deal with Navier-Stokes equations. In [5], RayleighBénard convection process for optimal control design on a POD based dynamic model is considered. Practically, this paper approaches the control problem from an observer based state reconstruction and state feedback point of view and the very role of the observer is the elimination of the noise corrupting the snapshots read from the infinite dimensional process.

This paper is organized as follows. In the second section, we present the POD and related modeling strategy, following this we focus on the design and analysis of the LD observer and the feedback control design is explained in the fourth section. The concluding remarks are given at the end of the paper.

\section{The Process AND LD Modeling By POD}

Consider the ensemble $U_{i}(x, y), i=1,2, \ldots, N_{s}$, where $N_{s}$ is the number of elements. Every element of this set corresponds to a snapshot observed from a process, say for example the 2D heat flow with initial and boundary conditions,

$$
\begin{aligned}
& u_{t}(x, y, t)=c^{2}\left(u_{x x}(x, y, t)+u_{y y}(x, y, t)\right) \\
& u(x, 0, t)=f_{1}(x) \gamma_{1}(t), u(1, y, t)=f_{2}(y) \gamma_{2}(t), \\
& u(x, 1, t)=f_{3}(x) \gamma_{3}(t), u(0, y, t)=f_{4}(y) \gamma_{4}(t), \\
& u(x, y, 0)=0 \quad \forall(x, y),
\end{aligned}
$$

where, $c$ is the constant thermal diffusivity parameter that is known, $f_{i}(\cdot)$ s are the gains effective along the boundary segments, and the subscripts $x, y$ and $t$ refer to the partial differentiation with respect to $x, y$ and time, respectively. The continuous time process takes place over the physical domain $\Omega:=\{(x, y) \mid(x, y) \in[0,1] \times[0,1]\}$ and the solution is obtained on a spatial grid denoted by $\Omega_{d}$, which describes the coordinates of the pixels of every snapshot in the ensemble. The entities described over $\Omega_{d}$ are matrices in $\mathbb{R}^{N_{y} \times N_{x}}$. The cost function denoted by $J$ is given by 


$$
J=\int_{0}^{\infty}\left(r \gamma_{1}^{2}(t)+w \int_{0}^{1} \int_{0}^{1}\left(u-u_{d}\right)^{2} \mathrm{~d} x \mathrm{~d} y\right) \mathrm{d} t,
$$

where $u_{d}(x, y, t)$ is the desired profile (thermal distribution) for $u(x, y, t)$ and $r, w$ are positive weighting coefficients. Note that the control problem studied here is to minimize the above cost by altering the input $\gamma_{1}(t)$ appropriately. It will later be clarified that the entries $\gamma_{2}(t), \gamma_{3}(t)$ and $\gamma_{4}(t)$ are utilized for the disturbance entries. The goal is to find an orthonormal basis set letting us to write the solution as

$$
u(x, y, t)=\sum_{i=1}^{R_{L}} \alpha_{i}(t) \Phi_{i}(x, y),
$$

where $\alpha_{i}(t)$ is the $i^{\text {th }}$ temporal mode, $\Phi_{i}(x, y)$ is the $i^{\text {th }}$ spatial function (basis functions or the eigenfunctions) defined over $\Omega, R_{L}$ is the number of independent basis functions that can be synthesized from the given ensemble, or equivalently that spans the space described by the ensemble. If the basis set $\left\{\Phi_{i}(x, y)\right\}_{i=1}^{R_{L}}$ is an orthonormal set, Galerkin projection yields the autonomous Ordinary Differential Equations (ODEs) directly. The POD procedure utilized in this study is described in [5] in detail.

Fundamental Assumption: The majority of works dealing with POD and model reduction applications presume that the flow (the solution of the PDE process) is dominated by coherent modes. Because of the dominance of coherent modes, the typical spread of the eigenvalues of the correlation matrix turns out to be logarithmic and the terms decay very rapidly in magnitude. This fact enables us to assume that a reduced order representation, say with $M<R_{L}$ modes can also be written as an equality

$$
u(x, y, t)=\sum_{i=1}^{M} \alpha_{i}(t) \Phi_{i}(x, y),
$$

and the reduced order model is derived under the assumption that (4) satisfies the governing PDE in (1), (See [5], [3], [6], [7]). Unsurprisingly, such an assumption results in a model having uncertainties, however, one should keep in mind that the goal is to find a model, which matches the infinite dimensional system in some sense of approximation with typically $M \ll R_{L} \leq N_{s}$, where $R_{L}$ denotes the rank of the correlation matrix $L$. To represent how good such an expansion is, a percent energy measure is defined as follows

$$
E=\frac{\sum_{i=1}^{M} \lambda_{i}}{\sum_{i=1}^{R_{L}} \lambda_{i}} \times 100 \%,
$$

where the tendency of $E \rightarrow 100 \%$ means that the model captures the dynamical information contained in the snapshots well. Conversely, an insufficient model will be obtained if $E$ is far below $100 \%$.

The second part of the fundamental assumption emphasizes the discretization of the PDE. Since the numerical solver computes the behavior of the process over a grid having finite number of pixels, we assume that the solution obtained over the computational grid is descriptive enough to admit the solution as a snapshot from the process.

In the next section, we demonstrate how the boundary condition is transformed to an explicit control input in the autonomous set of ODEs.

Theorem 2.1: The cost function in (2) is equivalent to (6) under the fundamental assumption.

$$
J=\int_{0}^{\infty}\left(w e^{\mathrm{T}} e+r \gamma_{1}^{2}(t)\right) \mathrm{d} t,
$$

where $e(t):=\alpha(t)-\alpha_{d}(t)$ and the desired profile is $u_{d}(x, y, t)=\sum_{i=1}^{M} \alpha_{d i}(t) \Phi_{i}(x, y)$.

The proof of Theorem 2.1 is by direct substitution of (4) and $u_{d}(x, y, t)=\sum_{i=1}^{M} \alpha_{d i}(t) \Phi_{i}(x, y)$ into (6) and the utilization of the orthonormality properties of the basis functions.

In the order reduction phase, we need to obtain the autonomous ODE model first. Towards this goal, if (4) is a solution to the PDE in (1), then it has to satisfy the PDE. Substituting (3) into (1) with the fundamental assumption yields

$$
\sum_{i=1}^{M} \dot{\alpha}_{i}(t) \Phi_{i}(x, y)=c^{2} \sum_{i=1}^{M} \alpha_{i}(t) \Psi_{i}(x, y),
$$

where $\Psi_{i}(x, y)=\frac{\partial^{2} \Phi_{i}(x, y)}{\partial x^{2}}+\frac{\partial^{2} \Phi_{i}(x, y)}{\partial y^{2}}$. Taking the inner product of both sides with $\Phi_{k}(x, y)$ and remembering $\left\langle\Phi_{i}(x, y), \Phi_{k}(x, y)\right\rangle_{\Omega}=\delta_{i k}$ with $\delta_{i k}$ being Kronecker delta yields

$$
\dot{\alpha}_{k}(t)=c^{2} \sum_{i=1}^{M} \alpha_{i}(t)\left\langle\Phi_{k}(x, y), \Psi_{i}(x, y)\right\rangle_{\Omega},
$$

Defining $\zeta_{k}$ as the entity in $\Omega_{d}$ corresponding to the entity $\Psi_{k}$ in $\Omega$, one could rewrite (8) as

$$
\dot{\alpha}_{k}(t)=c^{2} \sum_{i=1}^{M} \alpha_{i}(t)\left\langle\phi_{k}, \zeta_{i}\right\rangle_{\Omega_{d}} .
$$

Let $\mathcal{A}, \mathcal{B} \in \mathbb{R}^{N_{y} \times N_{x}}$. Define the inner product seen in (9) as $\mathcal{A} \star \mathcal{B}:=\frac{1}{N_{s}} \sum_{i=1}^{N_{y}} \sum_{j=1}^{N x} \mathcal{A}(i, j) \mathcal{B}(i, j)$ The equation in (9) can be written explicitly by using $\star$ operator as

$$
\dot{\alpha}_{k}(t)=c^{2} \sum_{i=1}^{M} \alpha_{i}(t)\left(\phi_{k}(x, y) \star \zeta_{i}(x, y)\right) .
$$

Notice that $\star$ operator can be applied over nonoverlapping subdomains of $\Omega_{d}$. This lets us separate the entries corresponding to boundaries without modifying the values of $\phi_{k}(x, y) \star \zeta_{i}(x, y)$ as seen in (11),

$$
\begin{aligned}
\dot{\alpha}_{k}(t)=\quad & c^{2} \sum_{i=1}^{M} \alpha_{i}(t)\left(\phi_{k}(x, 0) \star \zeta_{i}(x, 0)+\phi_{k}(1, y) \star \zeta_{i}(1, y)+\right. \\
& \left.\phi_{k}(x, 1) \star \zeta_{i}(x, 1)+\phi_{k}(0, y) \star \zeta_{i}(0, y)\right)+ \\
& c^{2} \sum_{i=1}^{M} \alpha_{i}(t)\left(\phi_{k}^{\circ}(x, y) \star \zeta_{i}^{\circ}(x, y)\right) .
\end{aligned}
$$


In above, $\phi_{k}^{\circ}(x, y)$ denotes a matrix that is obtained when the boundary elements of $\phi_{k}(x, y)$ are removed. The $k^{\text {th }}$ component of the first summation above, which is obtained when $i=k$, can be separated from the expression and we obtain (12), which let us embed the boundary conditions into the expression,

$$
\begin{aligned}
& \dot{\alpha}_{k}(t)=c^{2} \alpha_{k}(t)\left(\phi_{k}(x, 0) \star \zeta_{k}(x, 0)+\phi_{k}(1, y) \star \zeta_{k}(1, y)+\right. \\
& \left.\phi_{k}(x, 1) \star \zeta_{k}(x, 1)+\phi_{k}(0, y) \star \zeta_{k}(0, y)\right)+ \\
& c^{2} \sum_{i=1}^{M} \alpha_{i}(t)\left(1-\delta_{i k}\right)\left(\phi_{k}(x, 0) \star \zeta_{i}(x, 0)+\right. \\
& \phi_{k}(1, y) \star \zeta_{i}(1, y)+\phi_{k}(x, 1) \star \zeta_{i}(x, 1)+ \\
& \left.\phi_{k}(0, y) \star \zeta_{i}(0, y)\right)+c^{2} \sum_{i=1}^{M} \alpha_{i}(t)\left(\phi_{k}^{\circ}(x, y) \star \zeta_{i}^{\circ}(x, y)\right)
\end{aligned}
$$

At this stage of the modeling, we need to paraphrase the boundary conditions in such a way that the final expression above can be incorporated with these conditions. The underlying idea is straightforward: If (4) is a solution, then is must be satisfied at the boundaries as well. This lets us write the following

$$
\sum_{i=1}^{M} \alpha_{i}(t) \phi_{i}(x, 0)=f_{1}(x) \gamma_{1}(t)
$$

which can be paraphrased as

$$
\alpha_{k}(t) \phi_{k}(x, 0)=f_{1}(x) \gamma_{1}(t)-\sum_{i=1}^{M}\left(1-\delta_{i k}\right) \alpha_{i}(t) \phi_{i}(x, 0) \text {. }
$$

The expression above can be inserted into the first line of (12) and we explicitly see $\gamma_{1}(t)$ in our expression. Likewise, repeating the same arrangements for the other three edges (the boundaries), we end up with

$$
\begin{aligned}
\dot{\alpha}_{k}(t)= & c^{2}\left(f_{1}(x) \star \zeta_{k}(x, 0)\right) \gamma_{1}(t)+c^{2}\left(f_{2}(y) \star \zeta_{k}(1, y)\right) \gamma_{2}(t)+ \\
& c^{2}\left(f_{3}(x) \star \zeta_{k}(x, 1)\right) \gamma_{3}(t)+c^{2}\left(f_{4}(y) \star \zeta_{k}(0, y)\right) \gamma_{4}(t)+ \\
& c^{2} \sum_{i=1}^{M} \alpha_{i}(t)\left(\phi_{k} \star \zeta_{i}\right. \\
& -\phi_{i}(x, 0) \star \zeta_{k}(x, 0)-\phi_{i}(1, y) \star \zeta_{k}(1, y) \\
& \left.-\phi_{i}(x, 1) \star \zeta_{k}(x, 1)-\phi_{i}(0, y) \star \zeta_{k}(0, y)\right),
\end{aligned}
$$

which can be written compactly as

$$
\dot{\alpha}(t)=A \alpha(t)+B \Gamma(t)
$$

where $\alpha(t)=\left(\begin{array}{llll}\alpha_{1}(t) & \alpha_{2}(t) & \ldots & \left.\alpha_{M}(t)\right)^{\mathrm{T}},\end{array} \Gamma(t)=\right.$ $\left(\gamma_{1}(t) \gamma_{2}(t) \gamma_{3}(t) \gamma_{4}(t)\right)^{\mathrm{T}}$ and

$$
\begin{aligned}
A_{k i}= & c^{2}\left(\phi_{k}(x, y) \star \zeta_{i}(x, y)-\phi_{i}(x, 0) \star \zeta_{k}(x, 0)\right. \\
& -\phi_{i}(1, y) \star \zeta_{k}(1, y)-\phi_{i}(x, 1) \star \zeta_{k}(x, 1) \\
& \left.-\phi_{i}(0, y) \star \zeta_{k}(0, y)\right),
\end{aligned}
$$

and the $k^{\text {th }}$ row of the input matrix is

$$
\begin{aligned}
B_{k}= & c^{2}\left(f_{1}(x) \star \zeta_{k}(x, 0)\left|f_{2}(y) \star \zeta_{k}(1, y)\right|\right. \\
& \left.f_{3}(x) \star \zeta_{k}(x, 1) \mid f_{4}(y) \star \zeta_{k}(0, y)\right) .
\end{aligned}
$$

This result practically lets us have a non-autonomous linear dynamical model for the infinite dimensional process in (1), which is aimed to be controlled through the boundaries.

A natural question is whether this model recovers the unforced dynamics, which is obtained by setting $\gamma_{j}(t)=0$ for all four edges, and the steady state dynamics obtained when $\dot{\alpha}(t)=0$. Straightforward manipulations will show that the dynamics in (8) is recovered in both cases.

As seen clearly, once the finite dimensional model for an infinite dimensional process is obtained, one might implement an observer to obtain a useful state information and a boundary controller to minimize the cost in (2).

\section{Design AND ANALYSis of The ObSERVER}

First we demonstrate that a PDE observer can be proposed and the stability is maintained. Consider the following process

$$
\begin{aligned}
v_{t}(x, y, t) & =c^{2}\left(v_{x x}(x, y, t)+v_{y y}(x, y, t)\right)+\mathcal{K}(u-v) \\
v(x, 0, t) & =f_{1}(x) \gamma_{1}(t), \quad v(1, y, t)=f_{2}(y) \gamma_{2}(t) \\
v(x, 1, t) & =f_{3}(x) \gamma_{3}(t), \quad v(0, y, t)=f_{4}(y) \gamma_{4}(t),
\end{aligned}
$$

where $\mathcal{K}>0$ stands for the observer gain and we choose the following Lyapunov function candidate to study the stability

$$
V=\frac{1}{2} \int_{0}^{1} \int_{0}^{1}(u-v)^{2} \mathrm{~d} x \mathrm{~d} y
$$

and evaluate its time derivative as seen below,

$$
\begin{aligned}
\dot{V} & =\int_{0}^{1} \int_{0}^{1}(u-v)\left(u_{t}-v_{t}\right) \mathrm{d} x \mathrm{~d} y \\
& =-2 \mathcal{K} V+c^{2} \int_{0}^{1} \int_{0}^{1} z\left(z_{x x}+z_{y y}\right) \mathrm{d} x \mathrm{~d} y,
\end{aligned}
$$

where $z:=u-v$. It is straightforward to show that 


$$
\begin{aligned}
\int_{0}^{1} \int_{0}^{1} z z_{x x} \mathrm{~d} x \mathrm{~d} y & =\int_{0}^{1}\left(\left.z z_{x}\right|_{x=0} ^{x=1}-\int_{0}^{1} z_{x}^{2} d x\right) \mathrm{d} y \\
& =-\int_{0}^{1} \int_{0}^{1} z_{x}^{2} \mathrm{~d} x \mathrm{~d} y
\end{aligned}
$$

which is due to the fact that $z(1, y, t) z_{x}(1, y, t)-$ $z(0, y, t) z_{x}(0, y, t)=0$ as $z(1, y, t)=u(1, y, t)-$ $v(1, y, t)=f_{2}(y) \gamma_{2}(t)-f_{2}(y) \gamma_{2}(t)=0$ and $z(0, y, t)=$ $u(0, y, t)-v(0, y, t)=f_{2}(y) \gamma_{2}(t)-f_{2}(y) \gamma_{2}(t)=0$. Likewise we have

$$
\int_{0}^{1} \int_{0}^{1} z z_{y y} \mathrm{~d} x \mathrm{~d} y=-\int_{0}^{1} \int_{0}^{1} z_{y}^{2} \mathrm{~d} x \mathrm{~d} y
$$

Under these conditions, (21) becomes

$$
\dot{V}=-2 \mathcal{K} V-c^{2} \int_{0}^{1} \int_{0}^{1}\left(z_{x}^{2}+z_{y}^{2}\right) \mathrm{d} x \mathrm{~d} y<0 .
$$

Since $\dot{V}<0$ is guaranteed, $v(x, y, t) \rightarrow u(x, y, t)$ as $t \rightarrow \infty$, i.e. $v(x, y, t)$ reconstructs $u(x, y, t)$ in a globally exponentially stable fashion. If the process state $(u(x, y, t))$ is noisy, the observer filters out the noise and enables us to utilize the process state in the closed loop control system design.

The question here is whether this observer is useful for feedback control purposes. Towards this goal, recall the fundamental assumption, stating that the $M$-term summation can be assumed as the true solution and the error is negligible. This lets us write $v(x, y, t)=\sum_{i=1}^{M} \beta_{i}(t) \Phi_{i}(x, y)$, i.e. the dynamical constituents of the observer are projected onto the spatial eigenfunctions derived in the modeling stage, and this lets us have $M$ dimensional observer state, $\beta(t)$. Substituting above expression of $v(x, y, t)$ into the PDE in (19) yields

$$
\begin{aligned}
& \sum_{i=1}^{M} \dot{\beta}_{i}(t) \Phi_{i}(x, y)=c^{2} \sum_{i=1}^{M} \beta_{i}(t) \Psi_{i}(x, y)+ \\
& \mathcal{K} u(x, y, t)-\mathcal{K} \sum_{i=1}^{M} \beta_{i}(t) \Phi_{i}(x, y),
\end{aligned}
$$

where $\beta_{i}(t)$ is the temporal part corresponding to spatial eigenbasis $\Phi_{i}(x, y)$. Taking the inner product of both sides with $\Phi_{k}(x, y)$ lets us obtain (26),

$$
\dot{\beta}_{k}(t)=c^{2} \sum_{i=1}^{M} \beta_{i}(t)\left\langle\Phi_{k}(x, y), \Psi_{i}(x, y)\right\rangle_{\Omega}+\mathcal{K} \sigma(t) .
$$

where $\sigma(t)=\left(\begin{array}{llll}\sigma_{1}(t) & \sigma_{2}(t) & \ldots & \sigma_{M}(t)\end{array}\right)^{\mathrm{T}}$ and

$$
\begin{aligned}
\sigma_{k}(t)= & \left\langle u(x, y, t), \Phi_{k}(x, y)\right\rangle_{\Omega} \\
& -\sum_{i=1}^{M} \beta_{i}(t)\left\langle\Phi_{k}(x, y), \Phi_{i}(x, y)\right\rangle_{\Omega} \\
= & \left\langle U_{t}, \phi_{k}\right\rangle_{\Omega_{d}}-\beta_{k}(t),
\end{aligned}
$$

where $U_{t} \in \Omega_{d}$ is the snapshot of the process at time $t$. Following the modeling procedure discussed before, we end up with the LD observer given below

$$
\dot{\beta}(t)=A \beta(t)+B \Gamma(t)+\mathcal{K} \sigma(t),
$$

where $A$ and $B$ have been defined in (16)-(18). One should note that the computation of the term $\sigma_{k}(t)=\left\langle U_{t}, \phi_{k}\right\rangle_{\Omega_{d}}-$ $\beta_{k}(t)$ entails an infinite dimensional output feedback from the process, $U_{t}$, which is projected onto the eigenbasis, $\phi_{k}$, thereby forming a finite dimensional state information that is the counterpart of $\alpha_{k}(t)$. Clearly, $\sigma(t)$ can be interpreted as an equivalent measure for the state error under the fundamental assumption.

A last remark in this section is about meaning of the Lyapunov function in (20). Under the presence of the fundamental assumption, one can write the equalities in (29), which tells us that the infinite dimensional form of the Lyapunov function is mapped to a well-known Lyapunov function in $\mathbb{R}^{M}$. Therefore the proof of negative definiteness of one would clearly imply the other as long as the fundamental assumption holds true. In the next section, we consider the boundary control of the system by using the state information from the LD observer.

$$
\begin{aligned}
V_{o} & =\frac{1}{2} \int_{0}^{1} \int_{0}^{1}(u-v)^{2} \mathrm{~d} x \mathrm{~d} y \\
& =\frac{1}{2} \int_{0}^{1} \int_{0}^{1}\left(\sum_{i=1}^{M} \alpha_{i} \Phi_{i}-\sum_{i=1}^{M} \beta_{i} \Phi_{i}\right)^{2} \mathrm{~d} x \mathrm{~d} y \\
& =\frac{1}{2} \int_{0}^{1} \int_{0}^{1}\left(\sum_{i=1}^{M} e_{i} \Phi_{i}\right)^{2} \mathrm{~d} x \mathrm{~d} y \\
& =\frac{1}{2} e^{\mathrm{T}} e .
\end{aligned}
$$

\section{LinEAR QUADRATIC OPTIMAL BOUNDARY CONTROL OF THE 2D HEAT FLOW}

In this paper, we study the following scenario. As illustrated in Fig. 1, the inputs $\gamma_{2}, \gamma_{3}$ and $\gamma_{4}$ are the entries of external disturbances while $\gamma_{1}$ is reserved for the control signal. We partition the matrix $B$ as $B=\left(\begin{array}{ll}B_{c} & B_{d}\end{array}\right)$, where $B_{c}$ is $M \times 1$ vector and $B_{d}$ is an $M \times 3$ matrix.

At this stage, one needs to know the structural properties of the state space realization $\left(A, B_{c}\right)$ at hand, e.g. we need to know whether the states feel the control signal. For this reason, we have checked the rank of the matrix pencil $\left(\xi I-A \mid B_{c}\right)$, where $\xi$ is an eigenvalue of $A$ and 
have seen that for all eigenvalues of $A$, the rank of the matrix pencil is equal to $M=8$. Expectedly, the rank of the controllability grammian $\int_{0}^{\infty} e^{A t} B_{c} B_{c}^{*} e^{A^{*} t} \mathrm{~d} t$ is equal to $M=8$ but the rank of the controllability matrix ( $B_{c} \quad A B_{c} \quad A^{2} B_{c} \ldots A^{M-1} B_{c}$ ) is equal to 6 . We attribute this conflicting result to the numerical problems associated to the POD algorithm. Furthermore, this emphasizes that not all thermal profiles are realizable via feedback control. If we remember the issues in choosing $M$, it becomes clear that as $M$ gets larger, the reconstruction gets better yet the POD starts dealing with quickly changing signals with the same $\Delta x$ and $\Delta y$. This inevitably introduces some uncertainty into the numerical content on the matrices $A$ and $B$. According to the POD procedure, the modes are obtained in the order of dominance and this order is typically a logarithmic one. Therefore the modes having higher indices are less relevant to the essential dynamics and the associated states, though they may be uncontrollable, have negligibly small effects on the overall performance of the LD model. Since all potentially uncontrollable modes are stable, the system is stabilizable and the possible uncontrollability of these weak states is not an obstacle, which is the case we encounter in this study. A natural consequence of this discussion is that POD may not yield a useful model for every kind of PDE. Denote the interval for stable models by $M_{s \text { min }} \leq M \leq$ $M_{s \max }$ and interval for satisfactorily state reconstructing models by $M_{r \min } \leq M \leq M_{r \max }$. If the overlap of the intervals $\max \left(M_{s \min }, M_{r \text { min }}\right) \leq M \leq \min \left(M_{s \max }, M_{r \max }\right)$ is not empty and the dominant modes of the models are controllable, then the model can be used for feedback control system synthesis. The latter part of the statement contains some degrees of vagueness as what degree of dominance of an uncontrollable mode is absolutely a matter of the design problem in hand. But this discussion empirically shows that choosing the right number of modes depends upon many parameters. For the problem considered in this paper, we have $M_{s \min }=5$ and $M_{s \max }=\operatorname{rank}(L)=26, M_{r \min }=8$ and $M_{r \max }=\operatorname{rank}(L)=26$, therefore we have set $M=8$ for the simplest possible LD model.

The next issue is the selection of the desired behavior, which is expressed in terms of a set of temporal variables $\left(\alpha_{d}(t)\right)$ accompanied by the eigenbasis, which means that the target values of the state variables are available and accessible, i.e. $u_{d}(x, y, t)=\sum_{i=1}^{M} \alpha_{d i}(t) \Phi_{i}(x, y)$. A block diagram of the control system is depicted in Fig. 1, where $n_{i}(t)$ is the noise corrupting the observer inputs while $n_{o}(x, y, t)$ is a spatially continuous noise signal corrupting the process output, $u(x, y, t)$. According to this scenario, we would like to minimize the following cost function by exploiting the state information obtained through the LD observer;

$$
J=\int_{0}^{\infty} e(t)^{\mathrm{T}} W e(t)+r \gamma_{1}^{2}(t) \mathrm{d} t
$$

where $W=w I \in \mathbb{R}^{M \times M}$ is a positive definite weight matrix and $r \in \mathbb{R}$ is a positive scalar. $r$ and $w$ are the design parameters determining the relative importance between the state tracking precision and control effort. In our case the tracking precision is more important. The control law is given by

$$
\gamma_{1}=-r^{-1} B_{c}^{\mathrm{T}}\left(A^{\mathrm{T}}-Y B_{c} r^{-1} B_{c}^{\mathrm{T}}\right)^{-1} W \alpha_{d}-r^{-1} B_{c}^{\mathrm{T}} Y \beta
$$

where the matrix $Y$ is the solution of following Riccatti equation

$$
-Y A-A^{\mathrm{T}} Y-W+Y B_{c} r^{-1} B_{c}^{\mathrm{T}} Y=0_{M \times M}
$$

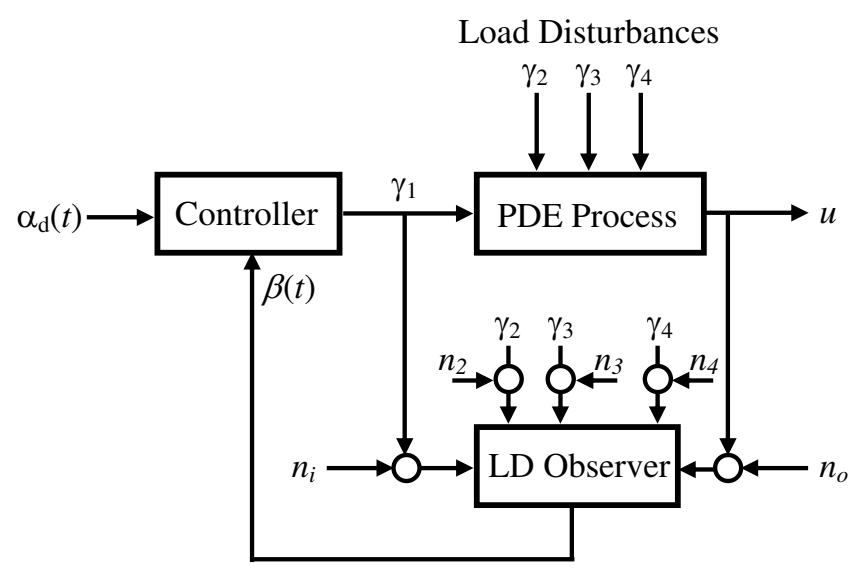

Fig. 1. Block diagram of the feedback control system

In order to compute the state tracking error, we adopt the following time varying reference model

$$
\dot{\alpha}_{d}(t)=c_{d}^{2}(t)\left(A \alpha_{d}(t)+B_{c} \mathcal{R}(t)\right)
$$

where the command signal for $0 \leq t \leq 1 \mathrm{sec}$. is $\mathcal{R}(t)=$ $\operatorname{sign}(\sin (10 \pi t))$ and for $1 \mathrm{sec} . \leq t \leq T=2 \mathrm{sec}$. is $\mathcal{R}(t)=$ $\sin (10 \pi t)$, which lets us see the performance of the closed loop control under different sorts of command signals. We adopt a time varying thermal diffusivity parameter in the reference model and incorporate this physical fact very easily as the quantity affects the corresponding model matrices $A$ and $B$ multiplicatively, and we set $c_{d}(t)=1+\frac{1}{2} \sin (4 \pi t)$.

One should note that the desired behavior is expressed in terms of the LD model therefore we know that the process is forced to follow the continuous-in-time snapshots implied by the reference model, which physically corresponds to a $2 \mathrm{D}$ heat flow with time varying thermal diffusivity parameter. For the goal of this paper, the state tracking precision is more important than the control effort. Therefore, in the simulations, we have chosen the LQ controller weights $W=$ $100 I_{M \times M}, r=1$, and the observer gain $\mathcal{K}=5$. These selections have let us obtain

$Y=\left(\begin{array}{llllllll}-0.8 & -7.78 & -0.98 & 0.56 & 7.0 & 9.2 & 1.1 & -1.2\end{array}\right)$ 
and in simulating the process, we have embedded the PDE solver into the Matlab/Simulink ${ }^{\circledR}$ environment. Two quantities have been investigated. First is $\sum_{k=1}^{M}\left(\left\langle U_{t}, \phi_{k}\right\rangle_{\Omega_{d}}-\beta_{k}(t)\right)^{2}=\|\sigma(t)\|_{2}^{2}$. The reason why we check this quantity is to figure out the observer's performance. The smaller the squared norm the closer the states of the observer to the equivalent process states. The second quantity is $\left\|\alpha_{d}(t)-\beta(t)\right\|_{2}^{2}$, which qualifies the controller performance. The smaller the quantity the better the state tracking in $\mathbb{R}^{M}$ and the better the tracking in $\Omega \times t \in[0, T]$. The latter implies the desire $u(x, y, t)$ tends to $u_{d}(x, y, t)$ through boundary excitations. In Fig. 2, the signals exciting the PDE process are illustrated. The signals shown in the figure have further been perturbed additively by zero mean Gaussian noise sequences having noise power 0.002 and the observer receives the noisy information from the process. This scenario is implemented to assess the performance of the observer under realistic operating conditions.
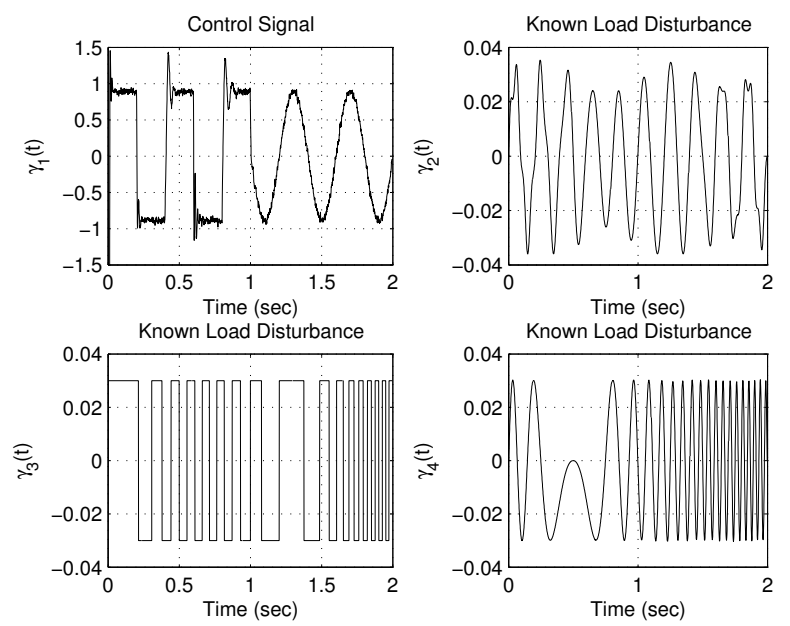

Fig. 2. Boundary signals exciting the PDE process

In Fig. 3, we illustrate how the designed observer captures the equivalent process states and how the LQ controller performs under the depicted simulation conditions. The upper two subplots are for the first 40 milliseconds of the simulation, where we see that the two performance measures have highly desirable characteristics. The bottom row of subplots depict the whole course of simulation in a logarithmic vertical axis. According to the results, the figure shows the fact that observer generates a useful state information and maintains the accuracy and stability despite very large initial errors between the $\alpha(t)$ and $\beta(t)$. It should be noted that parallel to the philosophy of POD, for dominant modes both accuracy and stability are substantially important while for the modes having high indices, the prime concern is only the maintenance of the stability as they have negligible effect on the overall behavior.

In Figs. 4 and 5, we illustrate several snapshots from the system. In both figures, the left columns depict the numerical solution from the process, the middle plots are the instanta-
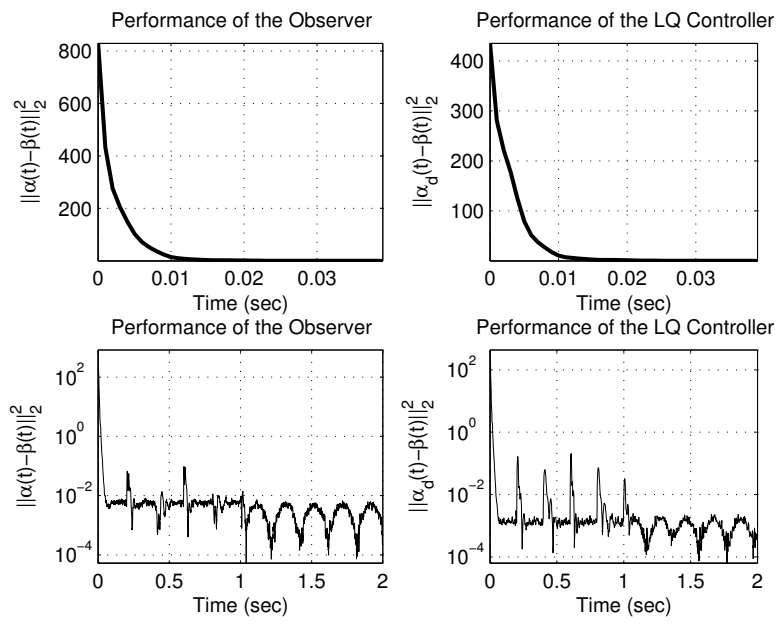

Fig. 3. Observer performance for reconstructing the equivalent process states and controller performance for state tracking

neous noisy signals (snapshots) fed to the observer and the right columns show the estimates obtained from the observer. Clearly the observer functions well in reconstructing the noiseless system response shown on the left column. since $T=2$ seconds, we sample the temporal evolution at integer multiples of 0.4 seconds and form the rows in Figs. 4 and 5. These results justify the claims of the paper. The next section summarizes the contributions of the paper to the subject area.

\section{CONCLUSiOnS}

This paper considers observer based LQ optimal boundary control of a particular type of $2 \mathrm{D}$ heat flow described in (1). The infinite dimensional process is approximated by a reduced order model by utilizing the POD technique with Galerkin projection. Having obtained the a model for the PDE process, an infinite dimensional version of the observer is proposed first and the necessary stability proof is given, then the finite dimensional form is postulated and the correspondence between them are investigated. The external excitations stimulate the process through the 1D segments of the physical domain $\Omega$, and the cost function is a quadratic one over an infinite time horizon. We therefore follow an optimal controller design scheme with the LD model and validate the controller with the PDE process. Quite rich thermal distributions are obtained due to the excitation through the boundary layer. According to the results obtained, we observed the following: 1) The LD model with control inputs separated appropriately functions well on some class of boundary conditions conditions. 2) The entire procedure of modeling and control system design is tightly dependent upon the fundamental assumption which is assumed to hold true for the considered process. 3) The simulation results have justified the claims and have shown that the selection of the mode number and the associated tradeoff emphasizes the case specificity. 4) The designed controller is able to reject the known load disturbances to an admissible extent. 5) The observer is able to alleviate initial errors, $n_{i}(t), n_{o}(x, y, t)$, 

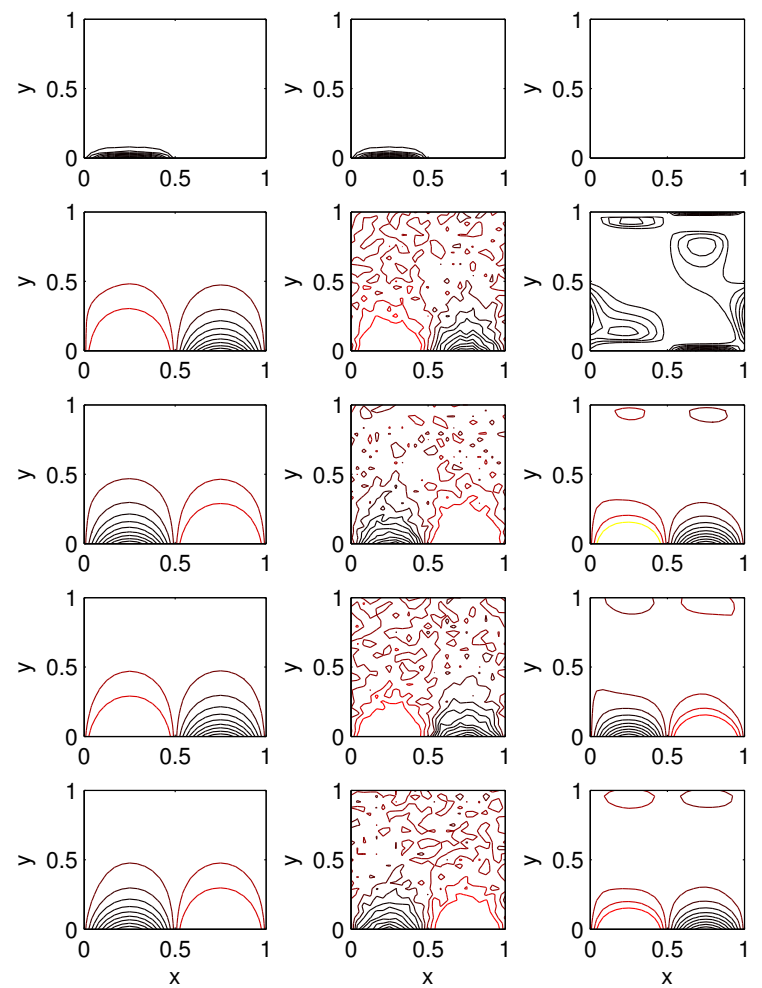

Fig. 4. Left: The snapshots from the process $(u(x, y, t))$, Middle: The noisy snapshots $\left(u(x, y, t)+n_{o}(x, y, t)\right)$ and Right: The response of the LD observer. The plots are for $0 \leq t \leq 1 \mathrm{sec}$.

and provide a reasonably clear state information that is to be used by the controller (See Figs. 4-5).

The results presented advance the subject area to the fact that POD based LD models can guide designing LD observers, and Lyapunov stability analysis help significantly in investigating the closed loop stability and classical tools can be used in the controller design. One fact needs emphasis, the fundamental assumption, i.e. the interchangeability of $v(x, y, t)=\sum_{i=1}^{R_{L}} \beta_{i}(t) \Phi_{i}(x, y)$ and $v(x, y, t)=$ $\sum_{i=1}^{M} \beta_{i}(t) \Phi_{i}(x, y)$ with $M<R_{L}$ indicate the information loss due to the negligence of the modes $M+1, M+2, \ldots, R_{L}$ is tolerable. This clearly highlights the case specificity in POD based modeling and control system synthesis works.

The future work in this field aims at implementing the modeling and control strategy for more demanding PDE processes.

\section{Acknowledgments}

The author would like to thank Prof. Hitay Özbay, Prof. Mo Samimy, Dr. James H. Myatt, Dr. J. DeBonis, Dr. Marco Debiasi, Dr. Russell C. Camphouse, Dr. Peng Yan, Xin Yuan and Edgar Caraballo for fruitful discussions in devising the presented work.
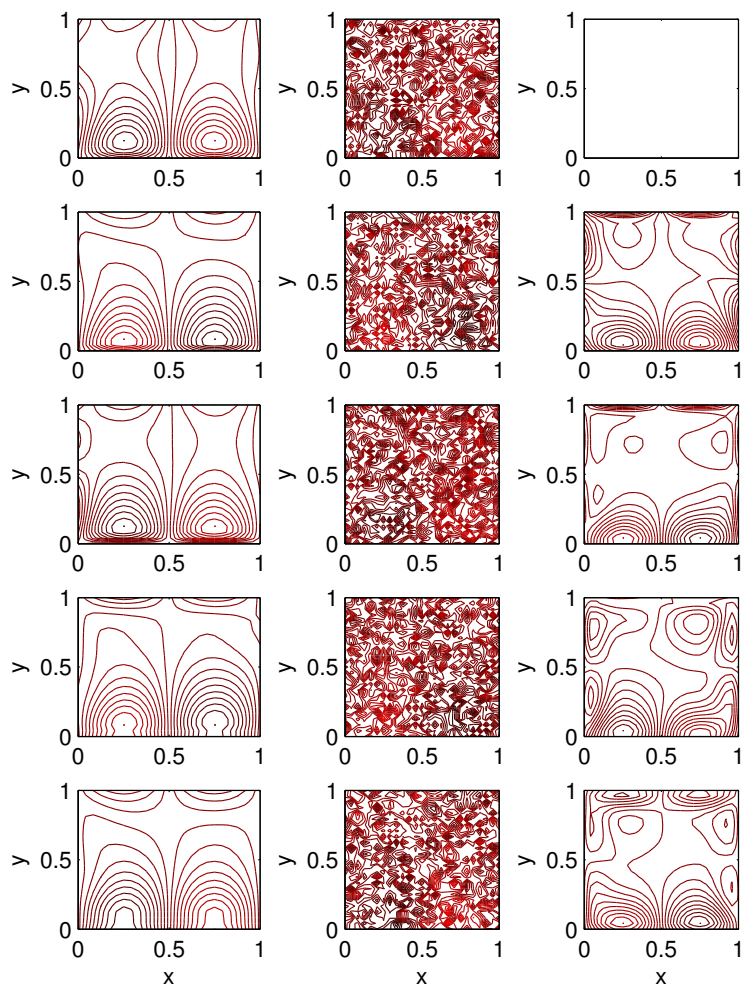

Fig. 5. Left: The snapshots from the process $(u(x, y, t))$, Middle: The noisy snapshots $\left(u(x, y, t)+n_{o}(x, y, t)\right)$ and Right: The response of the LD observer. The plots are for $1.4 \leq t \leq 2 \mathrm{sec}$.

\section{REFERENCES}

[1] Mizel, V.J. and Seidman T.I., "An Abstract Bang-bang Principle and Time-optimal Boundary Control of the Heat Equation," SIAM Journal on Control and Optimization, 35, 4, pp.1204-1216, 1997.

[2] Rösch A., "Identification of Nonlinear Heat Transfer Laws by Optimal Control," Numerical Functional Analysis and Optimization, 15, 3-4, pp.417-434, 1994.

[3] Ravindran, S.S., "A reduced order approach for optimal control of fluids using proper orthogonal decomposition," Int. Journal for Numerical Methods in Fluids, 34, pp.425-488, 2000.

[4] Singh S.N., Myatt J.H., Addington G.A., Banda S. and Hall J.K., "Optimal Feedback Control of Vortex Shedding Using Proper Orthogonal Decomposition Models," Trans. of the ASME: J. of Fluids Eng., 123, pp.612-618, 2001.

[5] Ly, H.V. and Tran, H.T., "Modeling and control of physical processes using proper orthogonal decomposition," Mathematical and Computer Modelling of Dynamical Systems, 33, pp.223-236, 2001.

[6] Rowley, C.W., Colonius, T, Murray, R.M., "Model reduction for compressible flows using POD and Galerkin projection," Physica DNonlinear Phenomena, 189 (1-2), pp.115-129, 2004.

[7] Rowley, C.W., "Model reduction for fluids, using balanced proper orthogonal decomposition," International Journal of Bifurcation and Chaos, 15 (3), pp.997-1013, 2005. 